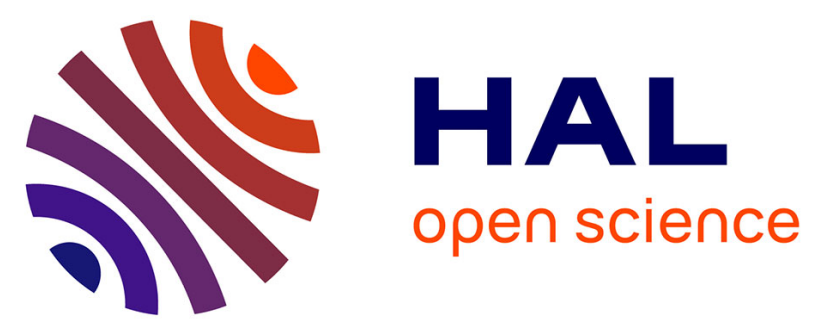

\title{
High-Precision Mid-Infrared Spectroscopy with a Widely Tuneable SI-Traceable Frequency-Comb-Stabilised QCL
}

Dang Bao An Tran, Rosa Santagata, Mathieu Manceau, Anne Cournol, Louis Lecordier, Bérengère Argence, Oliver Lopez, Sean K. Tokunaga, Fabrice Wiotte, Haniffe Mouhamad, et al.

\section{To cite this version:}

Dang Bao An Tran, Rosa Santagata, Mathieu Manceau, Anne Cournol, Louis Lecordier, et al.. HighPrecision Mid-Infrared Spectroscopy with a Widely Tuneable SI-Traceable Frequency-Comb-Stabilised QCL. 2019 44th International Conference on Infrared, Millimeter, and Terahertz Waves (IRMMWTHz), Sep 2019, Paris, France. pp.1-2, 10.1109/IRMMW-THz.2019.8874164 . hal-02410637

\section{HAL Id: hal-02410637 https://hal.science/hal-02410637}

Submitted on 15 Jan 2021

HAL is a multi-disciplinary open access archive for the deposit and dissemination of scientific research documents, whether they are published or not. The documents may come from teaching and research institutions in France or abroad, or from public or private research centers.
L'archive ouverte pluridisciplinaire HAL, est destinée au dépôt et à la diffusion de documents scientifiques de niveau recherche, publiés ou non, émanant des établissements d'enseignement et de recherche français ou étrangers, des laboratoires publics ou privés. 


\title{
High-Precision Mid-Infrared Spectroscopy with a Widely Tuneable SI- Traceable Frequency-Comb-Stabilised QCL
}

\author{
Dang Bao An $\operatorname{Tran}^{1}$, Rosa Santagata ${ }^{1}$, Mathieu Manceau ${ }^{1}$, Anne Cournol ${ }^{1}$, Louis Lecordier ${ }^{1}$, \\ Bérengère Argence ${ }^{1}$, Oliver Lopez ${ }^{1}$, Sean K. Tokunaga ${ }^{1}$, Fabrice Wiotte ${ }^{1}$, Haniffe Mouhamad ${ }^{1}$, Andrei \\ Goncharov $^{1}$, Michel Abgrall ${ }^{2}$, Yann Le Coq ${ }^{2}$, Hector Alvarez-Martinez ${ }^{2}$, Rodolphe Le Targat ${ }^{2}$, Won \\ Kiu Lee ${ }^{2}$, Dan Xu ${ }^{2}$, Paul-Eric Pottie ${ }^{2}$, Anne Amy-Klein ${ }^{1}$, and Benoît Darquié ${ }^{1}$ \\ ${ }^{1}$ Laboratoire de Physique des Lasers, Université Paris 13, Sorbonne Paris Cité, CNRS, Villetaneuse, France \\ ${ }^{2}$ LNE-SYRTE, Observatoire de Paris, Université PSL, CNRS, Sorbonne Université, Paris, France
}

\begin{abstract}
We report measurements of absolute frequencies of various polyatomic species around $10 \mu \mathrm{m}$, at level of accuracies ranging from $10 \mathrm{~Hz}$ to $10 \mathrm{kHz}$, using a widely tuneable SItraceable optical frequency comb-stabilized quantum cascade laser.
\end{abstract}

\section{INTRODUCTION}

$\mathrm{T}$ HERE is an increasing demand for precise molecular spectroscopy, in particular in the mid-infrared fingerprint window, whether it be for modelling our atmosphere, interpreting astrophysical spectra or testing fundamental physics. We present a high-resolution mid-infrared spectrometer traceable to primary frequency standards providing a unique combination of resolution, tuneability, detection sensitivity and frequency control.

\section{EXPERIMENTAL SETUP}

Fig. 1 shows the experimental setup, a mid-infrared spectrometer based on a widely tuneable, ultra-stable, and SItraceable quantum cascade laser (QCL) working at $10.3 \mu \mathrm{m}$.

This set up comprises a tuneable and ultra-stable optical local oscillator (OLO) based on a $1.54 \mu \mathrm{m}$ laser diode of frequency $v_{O L O}$. Two sidebands are generated in the OLO signal using an electro-optic modulator (EOM) driven by a home-made phasejump-free microwave synthesizer exhibiting a $9 \mathrm{GHz}$ continuous tuning range. The upper sideband of frequency $v_{O L O}+f_{E O M}$ (with $f_{E O M}$ the microwave frequency driving the EOM) is phase-locked to a $1.54 \mu \mathrm{m}$ remote optical frequency reference signal of frequency $v_{\text {ref }}$, using the phase-lock loop PLL1 shown in Fig. 1. This reference signal, generated at the French national metrological institute (LNE-SYRTE), is a 1.54 $\mu \mathrm{m}$ laser locked to an ultra-stable cavity and its absolute frequency is calibrated there against some of the world's best atomic clocks. It is then transferred to Laboratoire de Physique des Lasers through a 43-km long fibre link with active compensation of the propagation-induced phase noise. The OLO frequency is then given by

$$
v_{O L O}=v_{\text {ref }}-f_{E O M}-\Delta_{1},
$$

with $\Delta_{1}$ the frequency of the beat-note signal between $v_{O L O}+f_{E O M}$ and $v_{\text {ref }}$.

An optical frequency comb (OFC) with two outputs centred at 1.55 and $1.85 \mu \mathrm{m}$ is then used to transfer the spectral properties of the near-infrared reference signal to the QCL. We detect a beat-note signal between a comb tooth at frequency $p \times f_{\text {rep }}+f_{\text {ceo }}$ (with $f_{\text {rep }} \sim 250 \mathrm{MHz}$ the comb repetition rate, $p \sim 800000$ the comb mode integer, and $f_{c e o}$ the comb offset frequency) and $v_{O L O}$ on photodiode PD2 (see Fig. 1). After removing $f_{c e o}$, we obtain the resulting frequency beat-note signal at a frequency

$$
\Delta_{2}=v_{\text {OLO }}-p \times f_{\text {rep }} .
$$

The comb repetition rate is stabilized by locking $\Delta_{2}$ to a fixed radio-frequency signal (typically $150 \mathrm{MHz}$ ) using phase-lock loop PLL2.

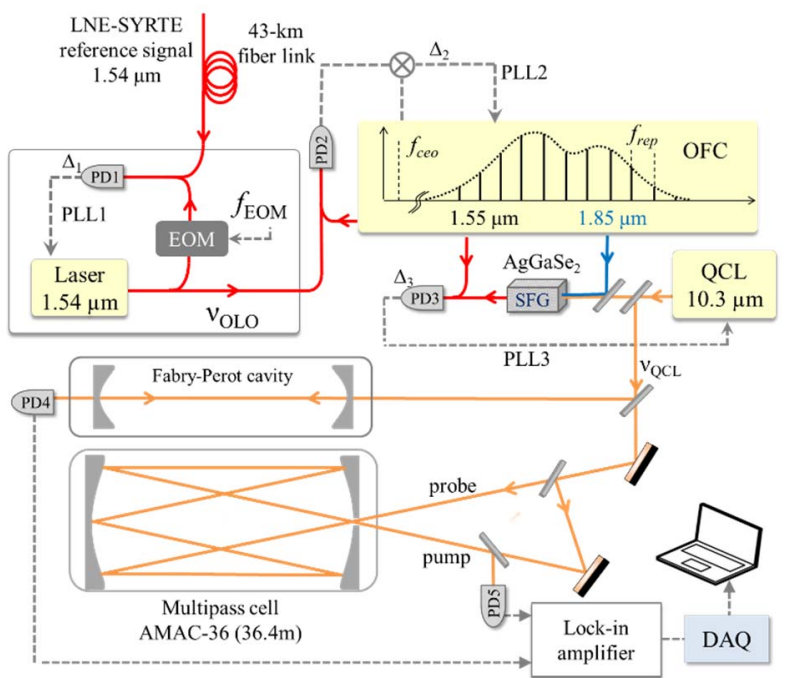

Fig. 1. Experimental setup. OFC: optical frequency comb; QCL: quantum cascade laser; PLL: phase-lock loop; EOM: electro-optic modulator; SFG: sum frequency generation, PD: photodiode, DDS: direct digital synthesizer, DAQ: acquisition card.

Finally, the QCL frequency is phase-locked to a high-order harmonic of the comb repetition rate. To this end, we perform sum-frequency generation between the comb output beam at $1.85 \mu \mathrm{m}$ (comb mode frequency $q \times f_{\text {rep }}+f_{\text {ceo }}$ with $q \sim 680000$ ) and the QCL beam, which results a shifted comb centred at $1.55 \mu \mathrm{m}$ with comb mode frequencies $q \times f_{\text {rep }}+f_{\text {ceo }}+v_{Q C L}$. In order to stabilize the QCL frequency, a beat-note signal at frequency $\Delta_{3}=(p-q) f_{\text {rep }}-v_{Q C L}$ between the shifted comb and the original comb is detected on photodiode PD3. The QCL frequency is finally stabilized by locking this beat-note signal to a fixed radio frequency signal (typically $11 \mathrm{MHz}$ ) using phase-lock loop PLL3. The QCL frequency is given by the following equation,

$$
v_{Q C L}=n \times f_{\text {rep }}-\Delta_{3}=\frac{n}{p}\left(v_{\text {ref }}-f_{E O M}-\Delta_{1}-\Delta_{2}\right)-\Delta_{3},
$$

where $n=p-q \sim 120000$.

The experimental setup therefore allows the stability and SI- 
traceability of the optical frequency reference, and the tuneability of the OLO to be transferred to the QCL. This results in a $\sim 0.1 \mathrm{~Hz}$ QCL linewidth, a stability below $10^{-15}$ from 0.1 to $10 \mathrm{~s}$ and an uncertainty on the absolute frequency below $4 \times 10^{-}$ ${ }^{14}[1,2]$. The spectral range covered by our spectrometer is $\sim 100$ $\mathrm{GHz}$ limited by the spectral window of the QCL used. Scanning the OLO carrier frequency over $9 \mathrm{GHz}$ allows the QCL frequency to be tuned over more than $1 \mathrm{GHz}$.

\section{RESULTS}

As illustrated in Fig. 1, we have used the apparatus to carry out ultra-high resolution spectroscopy of polyatomic molecules in a compact multipass absorption cell and few-meter-long Fabry-Perot cavities. We use frequency modulation to improve detection sensitivity.

In order to demonstrate the apparatus' potential for state-ofthe-art high-precision spectroscopic measurements, we have conducted saturated absorption spectroscopy of $\mathrm{OsO}_{4}$ using a Fabry-Perot cavity [1]. Fig. 2 shows a sub-Doppler spectrum of two rovibrational transitions of $\mathrm{OsO}_{4}$ averaged over ten up and down scans. It is recoded using frequency modulation and thirdharmonic detection. The QCL frequency is swept by scanning the frequency of the synthesizer used to phase-lock the QCL to the OFC. Typical experimental parameters are a pressure of $0.04 \mathrm{~Pa}$, an intra-cavity power of $50 \mu \mathrm{W}$, and a beam diameter of $6 \mathrm{~mm}$. The spectrum exhibits a peak-to-peak linewidth of $\sim 25 \mathrm{kHz}$ and a signal to noise ratio of 200 . The data is fitted to a combination of a third and a fifth derivative of a Lorentzian to account for the line shape deformation caused by the choice of frequency modulation parameters. The line-centre frequencies of these transitions and others were determined at a level of accuracy ranging from 20 to $40 \mathrm{~Hz}$ (see [1] for more details).

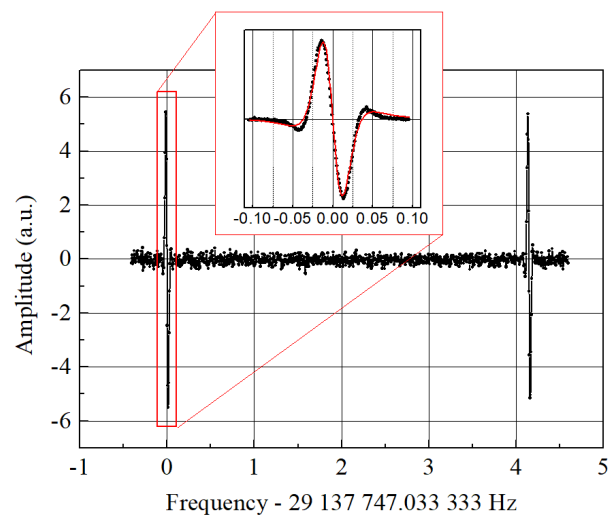

Fig. 2. Saturated absorption spectrum of $\mathrm{OsO}_{4}$ recorded using a Fabry-Perot cavity. Two lines are recorded over a span of $5 \mathrm{MHz}$ in steps of $5 \mathrm{kHz}$, using frequency modulation and third harmonic detection. Inset: spectrum of the reference line recorded over $200 \mathrm{kHz}$ in steps of $1 \mathrm{kHz}$. Taken from [1].

As illustrated in Fig. 3, we have also carried out saturated absorption spectroscopy of several rovibrational transitions of methanol using a multipass absorption cell. Typical experimental conditions are a pressure of $1 \mathrm{~Pa}$, an average intracell power of $\sim 1 \mathrm{~mW}$, a laser beam waist of $\sim 1 \mathrm{~mm}$ and firstharmonic detection. The QCL frequency is swept by scanning the EOM frequency and in turn the OLO carrier frequency. Fig. 3(b) displays a sub-Doppler spectrum of the $P(E, c o, 0,2,33)$ rovibrational line of methanol averaged over only one up and down scan. It exhibits a peak-to-peak linewidth of $\sim 400 \mathrm{kHz}$ and a signal-to-noise ratio of 310 . The solid yellow line is a fit to the data (see [2] for details of the line shape used). The linecentre frequency of the transition was determined with a statistical uncertainty of $\sim 1 \mathrm{kHz}$ and sub- $10 \mathrm{kHz}$ global uncertainty. Fig. 3(b) demonstrates the unprecedented continuous tuning capability resulting from using our widely tuneable and ultra-stable OLO. It shows a sub-Doppler spectrum of methanol around $971.565 \mathrm{~cm}^{-1}$ spanning $400 \mathrm{MHz}$ and exhibiting five rovibrational transitions of methanol [2].

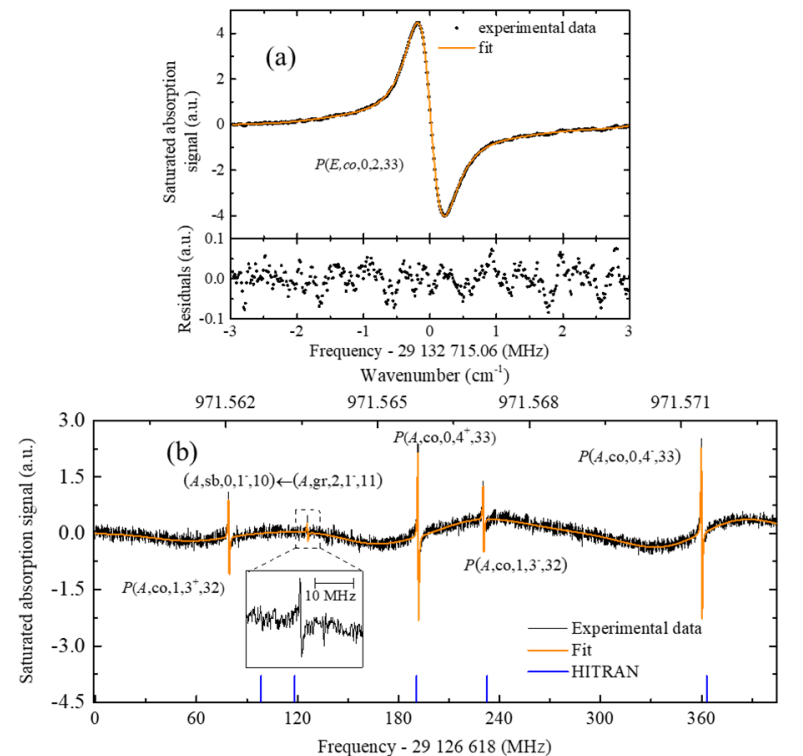

Fig. 3. (a) Saturated absorption spectrum of the $P(E, c o, 0,2,33)$ rovibrational transition of methanol, with fit and residuals. (b) Saturated absorption spectrum of methanol spanning $400 \mathrm{MHz}$. Taken from [2].

To conclude, we have developed an apparatus showing an unprecedented combination of wide tuneability, high sensitivity, and spectral resolution and used it to carry out saturated absorption spectroscopy. We have demonstrated record uncertainties ranging from $\sim 10 \mathrm{~Hz}$ to $10 \mathrm{kHz}$ on rovibrational absolute frequencies of various species of interest for space and atmospheric physics, and for testing fundamental physics, including osmium tetroxide, methanol, ammonia, trioxane... This confirms our apparatus' potential for our ongoing precise spectroscopic measurements with molecules [3].

\section{REFERENCES}

[1]. B. Argence, B. Chanteau, O. Lopez, D. Nicolodi, M. Abgrall, C. Chardonnet, C. Daussy, B. Darquié, Y. Le Coq, and A. Amy-Klein, "Quantum cascade laser frequency stabilization at the sub-Hz level," Nature Photonics, vol. 9, pp. 456-460, 2015.

[2]. R. Santagata, D.B.A. Tran, B. Argence, O. Lopez, S.K. Tokunaga, F. Wiotte, H. Mouhamad, A. Goncharov, M. Abgrall, Y. Le Coq, H. AlvarezMartinez, R. Le Targat, W.K. Lee, D. Xu, P.-E. Pottie, B. Darquié, and A AmyKlein, "High-precision methanol spectroscopy with a widely tunable SItraceable frequency-comb-based mid-IR QCL," Optica, vol. 6, pp. 411-423, 2019.

[3]. A. Cournol, M. Manceau, M. Pierens, L. Lecordier, D. B. A. Tran, R. Santagata, B. Argence, A. Goncharov, O. Lopez, M. Abgrall, Y. L. Coq, R. L. Targat, H. A. Martinez, W. K. Lee, D. Xu, P. E. Pottie, R. J. Hendricks, T. E. Wall, J. M. Bieniewska, B. E. Sauer, M. R. Tarbutt, A. Amy-Klein, S. K. Tokunaga, and B. Darquié, "A new experiment to test parity symmetry in cold chiral molecules using vibrational spectroscopy," Quantum Electronics vol 49, pp. 288-292, 2019. 